\title{
Reservation Arbitrated Access for Statistical Multiplexing Voice Traffic over Dual-Bus Metropolitan Area Networks
}

\author{
Henry C. B. Chan, Member, IEEE, and Victor C. M. Leung, Senior Member, IEEE
}

\begin{abstract}
Metropolitan area networks (MAN's) are well suited to serve as broadband multiplexers for asynchronous transfer mode (ATM) networks, to facilitate enterprise networking and to support future wireless personal communication systems. We propose and analyze a novel reservation arbitrated (RA) access method which provides isochronous voice transport over dual-bus MAN's while enabling statistical multiplexing among voice calls. In combination with a new cyclic capturing (CC) mechanism, RA access allows stations to capture and reserve isochronous voice channels in a fair and distributive manner. This paper presents the RA access protocol, derives an analytical model for general waste-free voice reservation protocols, and analyzes the performance of RA access by computer simulations validated by analytical calculations. To assess the actual voice quality, simulation results based on a real voice signal are also presented. Results indicate that RA access offers significant improvements in channel utilization, as compared to prearbitrated (PA) access, while providing an acceptable quality of service. Therefore, RA access offers an efficient voice transport mechanism for existing switched multimegabit data service (SMDS) networks employing the IEEE 802.6 protocol, as well as emerging ATM/MAN-based broadband networks.
\end{abstract}

Index Terms-Metropolitan area networks, reservation protocols, speech communication.

\section{INTRODUCTION}

$\mathbf{I}$ EEE 802.6 [1] is an international standard for metropolitan area networks (MAN's) based on a dual-bus architecture. In an IEEE 802.6 MAN (hereinafter referred as a MAN), communication nodes are connected to two unidirectional slotted buses, called bus A and bus B, each running at 155 $\mathrm{Mb} / \mathrm{s}$ (Fig. 1). The two buses support full duplex communications in opposite directions between any pair of nodes in the network. A special node called the head of bus (HOB) generates 53-octet (byte) time slots continuously at the head end of each bus for access by the downstream nodes. The IEEE 802.6 Standard supports asynchronous data transport

Manuscript received November 28, 1997; revised April 29, 1998. This work was supported in part by the Canadian Natural Sciences and Engineering Research Council under a Postgraduate Scholarship and Grant OGP0044286. This paper was presented in part at IEEE Globecom' 95.

H. C. B. Chan was with the Department of Electrical and Computer Engineering, The University of British Columbia, Vancouver, B.C. Canada V6T 1Z4 Canada. He is now with the Department of Computing, Hong Kong Polytechnic University, Hung Hom, Kowloon, Hong Kong.

V. C. M. Leung is with the Department of Electrical and Computer Engineering, The University of British Columbia, Vancouver, B.C. Canada V6T 1Z4 Canada.

Publisher Item Identifier S 0733-8716(99)00009-8.

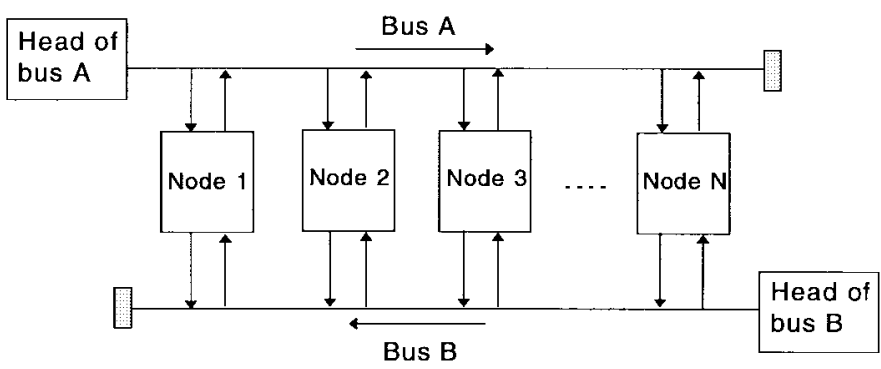

Fig. 1. Network configuration of IEEE 802.6 MAN.

by means of queue-arbitrated (QA) access employing the distributed-queue dual-bus (DQDB) protocol and connectionoriented isochronous data transport by means of prearbitrated (PA) access to preassigned isochronous channels identified by the virtual circuit identifier (VCI) of each PA slot. At present, MAN's have been deployed to provide customer access to the switched multimegabit data service (SMDS) [2]. Using the same packet (cell) size as broadband integrated services digital networks employing asynchronous transfer mode (ATM/BISDN's), MAN's are also expected to function as broadband multiplexers to facilitate the interconnection of various corporate and public networks with the emerging ATM/BISDN's, effectively serving as peripheral gathering networks of end-user traffic for transport over ATM/BISDN's [3], [4]. Furthermore, MAN's have also been proposed as distributed switches for future wireless personal communication networks to enable efficient call handoff management and distributed call processing [5], [6]. As voice will continue to account for a significant amount of traffic in the MANbased systems, the requirement of an efficient voice transport mechanism over MAN's is of primary importance.

While PA access is suitable for constant bit rate voice services, it is inefficient for transporting bursty packetized voice traffic as capacity needs to be preallocated to each call based on the peak bit rate. This is due to the alternating speech/silence intervals in voice conversations, i.e., on average, each party in a voice conversation talks during about only $40 \%$ of the time [7], [8]. Whereas transporting voice packets by QA access allows full statistical multiplexing, it suffers from access delay variations which necessitate the use of play-back buffers at the receivers. Due to the isochronous nature of voice, it is desirable to set up and tear down a PA connection on a per-talkspurt basis. However, signaling 
protocols which enable fast establishment/disconnection of PA connections do not exist. A reservation-based technique, i.e., a voice reservation protocol, should provide a more effective transport solution by enabling this operation. In recent years there has been many contention-based voice reservation protocols proposed for multiplexing voice with other variable bit rate traffic over various networks, such as the packet reservation multiple access (PRMA) [7] for wireless personal communication networks, the protocols proposed in [8] and [9] for local access networks (LAN's)/MAN's, and the adaptive digital access protocol for hybrid fiber-coax networks [10]. These protocols work in a similar manner, which allows the active voice stations to make reservations on a reverse channel and, based on the reservations, reserves sufficient slots in subsequent frames for each active voice station to transmit its packet. Although all of these reservation protocols allow statistical multiplexing, in some cases access may not be fair or may not be truly isochronous after a reservation is secured. In all cases, packet formats are not compatible with the slot format of the current MAN standard. While these protocols may be adapted for 802.6 MAN's, the distributive nature of MAN's should enable a more effective solution that minimizes the effects of reservation contentions while maintaining isochronous service.

Motivated by the above reservation protocols, we propose a new voice reservation protocol called reservation arbitrated (RA) access for isochronous voice traffic transport over dualbus MAN's. With RA access, the voice station acquires an RA slot when a user starts talking and, if successful, keeps the same slot in reservation in subsequent frames for the duration of the talkspurt. To maintain bounded transmission delay, packets that cannot be sent within a specific time limit are discarded. In fact, a rich family of RA access schemes can be found based on different methods of initial slot acquisition. In this paper, we consider the onepersistent and cyclic capturing (CC) methods. We derive an analytical model for general voice reservation protocols and analyze the performance of RA access by computer simulations validated by the analytical calculations. There have been many performance analyses on different packet voice systems in the literature [7]-[9], [11]-[13]. All of them assume a on-off voice source model and employ various statistical techniques for evaluating the clipping probability of the voice stations. In particular, [8], [9], and [11] are devoted to LAN's/MAN's. Compared to those, we not only evaluate the average clipping probability but also its upper and lower bound values, as well as the probability distribution of the number of clipped packets. Furthermore, we also simulate RA access using packets from a real voice conversation to assess the actual quality of a voice conversation. While the initial access of RA access is subject to possible packet losses, our results show that the system can be engineered to keep these degradations within acceptable levels. Compared to the reservation protocols proposed in [8] and [9], RA access can substantially improve access fairness by employing the $\mathrm{CC}$ mechanism and provide exact isochronous service (i.e., the same as PA access) once a reservation is secured. Its compatibility with the IEEE 802.6

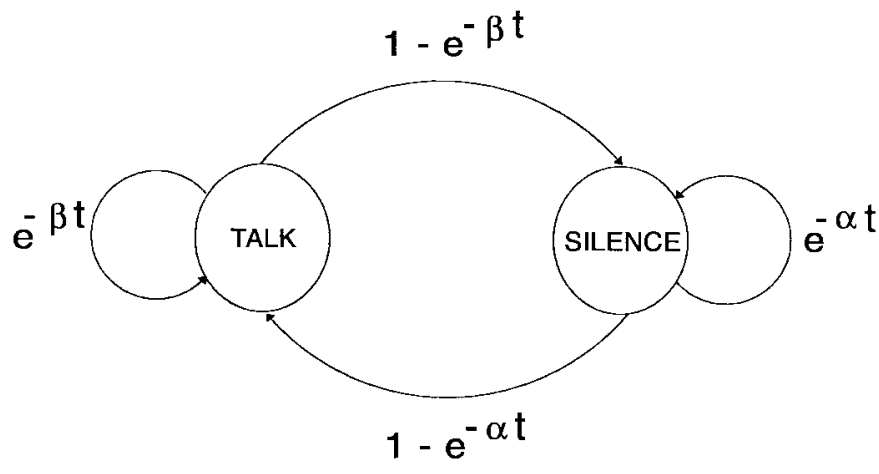

Fig. 2. Voice conversation model.

access control field (ACF) format also eases implementation over existing MAN's.

The organization of the paper is as follows. Section II gives the voice system model and the performance criteria on which performance analyzes in later sections are based. Section III explains the RA access protocol. Section IV presents an analytical model for general waste-free voice reservation protocols. Section V presents and discusses the analytical and simulation results. Section VI concludes the paper.

\section{Voice System Model ANd PeRformance CRITERIA}

Due to the symmetry of bus A and bus B, we shall consider traffic on bus A only. Throughout this paper, we assume that there are $N_{v}+1=51$ MAN nodes (i.e., $N=N_{v}+1$ in Fig. 1) with one voice station per node, each engaged in a call. As the $\left(N_{v}+1\right)$ th node does not have any packet to send on bus A, there are effectively $N_{v}=50$ voice stations. Each voice station alternates between talk (active) and silent (idle) periods, which are independent and exponentially distributed by means of $1 / \beta=1.5 \mathrm{~s}$ and $1 / \alpha=2.25 \mathrm{~s}$, respectively, for a voice activity factor of $r=(\alpha / \alpha+\beta)=40 \%$ as modeled by the bistate continuous time Markov process shown in Fig. 2 [14]. This model is widely used in the literature [7], [8], [11] for packet voice system analysis. During an active period, a voice station generates a 48-byte packet every $6 \mathrm{~ms}$ (assuming $64-\mathrm{Kb} / \mathrm{s}$ pulse code modulation coding). The packet is stored in the voice station's buffer before transmission. The buffer holds one packet, so that if a packet cannot be transmitted within one frame, it will be overwritten by a new packet and hence lost. This gives a bounded access delay of $6 \mathrm{~ms}$ for all successfully transmitted packets. A voice station does not transmit any packet during an idle period.

Note that with suitable choices of $\alpha$ and $\beta$ the bistate source model can effectively be used as the basic element for modeling packet video traffic. This had been verified by matching the average bit rate and autocovariance of experimental data from a video telephone scene [15] to the model. Hence, the RA access protocol and its analysis in this paper may also be extended for general variable bit rate isochronous traffic.

To evaluate voice performance of the system, the following performance criteria are used.

Clipping Probability: The clipping probability or, in general, packet loss probability, is defined as the fraction of generated packets being discarded, i.e., 1 - (number of 
successfully transmitted packets $\div$ number of packets generated). Previous research indicates that voice conversations can tolerate a small fraction of packet loss. To maintain acceptable voice quality, the average clipping probability should not exceed 0.01 according to various subjective tests [7], [16].

Clip Duration: The effect of consecutive packet losses is subjectively more severe than random packet losses. We define the clip duration as the duration of consecutive packet losses. The Campanella criteria [17] specify that, for acceptable voice quality, no more than $2 \%$ of all talkspurts should have clip durations greater than $50 \mathrm{~ms}$.

Utilization: The efficiency of a protocol is measured by the utilization of the allocated bandwidth, defined here as the fraction of slots actually carrying voice packets relative to the total number of slots allocated in the system for shared access by all voice stations. For PA access, the utilization would be fairly low, around $40 \%$, according to the above voice model.

Note that while access delay and access delay variations are also of interest for voice traffic, these two performance measures are less important in the case of RA access because the protocol guarantees a bounded initial access delay of 6 ms for each talkspurt and provides an isochronous transport service with no delay variations once a reservation is secured. The isochronous transport of each talkspurt ensures that no play-back buffering is required at the receiving voice station.

\section{THE RA ACCESS PROTOCOL}

As mentioned above, we shall consider packet transmissions on bus A only. A new slot type called the RA slot is introduced to complement PA and QA slots, as defined in the IEEE 802.6 Standard [1]. RA slots are accessible by all voice stations using the RA access protocol defined below. To ensure compatibility with the IEEE 802.6 Standard, RA slots have the same slot structure and bit assignments as the PA and QA slots, except for the (currently unused) reserved bits in the ACF. We propose using the reserved condition, SL_TYPE $=1$ and BUSY $=0$ to indicate an RA slot, and designate the two reserved bits in the ACF as the capture (CAP) bit and reserve (RES) bit used for RA access control, as shown in Table I. Note that the two bits indicating an RA slot introduce an extra slot processing delay of one bit-duration for each slot passing a node, as it is impossible to distinguish between an idle QA slot and an RA slot until the second bit (SL_TYPE) in the ACF is received, Nevertheless, we assume that this extra processing delay is acceptable and justified by the advantages of RA access. The proposed frame period is $f=6 \mathrm{~ms}$ such that a 48 -byte packet generated by $64-\mathrm{Kb} / \mathrm{s}$ voice coding fills up the payload of an RA/PA slot exactly once every frame. For compatibility with existing standards [1], the proposed 6ms (macro) frame can be considered as being made up of 48 (micro) frames of $125 \mu$ s duration. Assuming a bus data rate of $155 \mathrm{Mb} / \mathrm{s}$, each frame contains $F=2197$ slots, each holding a 53-byte packet (cell), plus some synchronization bits. The slots in each frame are numbered 1, 2, 3, .., 2197 and all nodes are synchronized to recognize the frame and slot boundaries.

The basic operation of RA access is explained as follows. A station starting a talkspurt first captures an available (idle)
TABLE I

Proposed ACF OF AN RA SLOT

\begin{tabular}{l|l|l|l|l|l|l|l}
\hline BUSY & SL_TYPE & PSR & CAP & RES & REQ0 & REQ1 & REQ2 \\
\hline 0 & 1 & 1 bit & 1 bit & 1 bit & 1 bit & 1 bit & 1 bit \\
\hline
\end{tabular}

RA slot $s$ (with CAP $=0$ ) on the forward bus by setting CAP $=1$ in slot $s$. The station then requests to reserve slot $s$ by marking the RES $=1$ in the corresponding slot $s^{*}$ on the reverse bus. The relationship between $s$ and $s^{*}$ will be addressed later. When HOB A receives the reservation indicated by RES $=1$ in slot $s^{*}$, it turns slot $s$ in each subsequent frame into a reserved RA slot, which is equivalent to a PA slot (SL_TYPE $=1$, BUSY = 1) with the VCI unmarked so that exact isochronous service can be provided to the station as in PA access. At the end of the talkspurt, the station discontinues the marking of the CAP bit in slot $s$ on the forward bus and the RES bit in $s^{*}$ on the reverse bus. Slot $s$ will be released by HOB A by reverting it to an idle RA slot, unless it is first captured by a downstream node using the cyclic release/capture mechanism described below.

In general, if an RA slot $s$ captured in frame $k$ cannot be turned into a reserved RA slot in frame $k+1$, due to propagation delay between the station and HOB A, access contention may occur because another voice station may still capture slot $s$ in frame $k+1$. This conflict can be resolved by the simple contention resolution method proposed in our preliminary work on RA access [18] in which the upstream voice station yields to the voice station furthest downstream. Nevertheless, if round trip delay is less than frame period $f$ it is possible to eliminate access contention by choosing $s^{*}$ appropriately such that the reservation conveyed by $s^{*}$ always reaches the HOB before it generates the corresponding slot $s$ in the next frame. For example, if $\mathrm{HOB}$ A and $\mathrm{B}$ generate slots in a synchronous manner (i.e., they both generate slot one at time zero) and the round trip delay is 1000 slots, it is not difficult to verify that the contention free condition can be satisfied if we ensure that

$$
s \oplus 500<s^{*}<s \oplus(F-1000)=s \oplus 1197
$$

where $\oplus$ denotes modulo $F$ addition. Note that with the above round trip delay of 1000 slots, the bus length is $560 \mathrm{~km}$, which well exceeds the expected coverage area of most MAN's. Consequently, it is quite feasible to avoid access contention by choosing $s$ and $s^{*}$ as stated above. For this reason, we will focus on the operation of contention-free RA access in this paper. More generally, the contention resolution method proposed in [18] can be used.

A family of RA access schemes can be found based on different methods of acquiring (capturing) the initial slot. The simplest capturing method is one-persistent capturing (1PC) in which only the respective HOB's are allowed to release the reserved slots and a requesting station always captures the first available idle RA slot. This follows the same concept as the voice reservation protocol in [8] and [9]. Although 1PC ensures no slot wastage, it is unfair with respect to the relative positions of the stations on the bus. To overcome the unfairness problem, we propose a new $\mathrm{CC}$ mechanism which is a simple 
enhancement of 1PC. The basic concept of RA access with CC is that all voice stations still capture the first available RA slots with probability one. When an active voice station becomes silent in frame $k$, however, (i.e., its packet buffer is empty) it immediately releases the reserved slot $s$ on bus A by setting $\mathrm{CAP}=0$ and stops marking the RES bit of corresponding slot $s^{*}$ on bus B. By doing so, first access to released slots will be given to the downstream stations in a round-robin manner, which prevents the upstream voice stations from preempting the downstream stations to obtain the reservations all the time. As shown by later simulation results, RA access with CC provides a reasonably fair service and yields performance close to the ideal waste-free voice reservation protocol.

It is also important to consider the robustness of the protocol, particularly in case the CAP/RES bit is corrupted (e.g., due to channel noise). While this event is highly unlikely in MAN's employing optical fiber media, should it occur, more than one station may incorrectly interpret that they have reserved the same slot and, consequently, some voice packets may be overridden by others. This problem will be rectified, however, when the involved stations return to the silent state and clear their reservations. This indicates the desired property that even if the protocol enters an incorrect state, it will eventually reenter a correct state, thus avoiding any deadlock or live-lock problem.

\section{AnAlytical Modeling of A WASTE-FreE Voice RESERVATION PROTOCOL}

This section presents an analytical model for a waste-free voice reservation protocol. A voice reservation protocol is defined as waste free if no slot is wasted during the slot acquisition (i.e., capturing) process. We shall validate the simulation results with the analytical computations of this ideal protocol because it is expected that the two results should be close to each other. These assumptions are made to facilitate the analysis.

1) A slot is effectively reserved when it is captured. In other words, no access contention occurs during the capturing process. Note that for RA access this can be realized under the typical coverage of a MAN as explained above.

2) Voice stations change state (active or idle) only at frame boundaries.

3) Each voice station may change state, at most, once at each frame boundary.

4) Talkspurts are much longer than the reservation delays so that a voice station starting a talkspurt can always capture and reserve a slot before the end of the talkspurt.

\section{A. Average Clipping Probability}

The average clipping probability is defined as the fraction of total packet loss relative to the total number of packets generated by all voice stations. For a waste-free voice reservation protocol, $\left(n-N_{s}\right)^{+}=\max \left\{0,\left(n-N_{s}\right)\right\}$ packets will be discarded in a frame when there are $n$ active voice stations and $N_{s}$ RA slots available per frame. As $B\left(N_{v}, n, r\right)$ gives the probability that $n$ of the $N_{v}$ voice stations are active at any time, where $B(X, Y, p)=\left(\begin{array}{l}X \\ Y\end{array}\right) p^{Y} \times(1-p)^{X-Y}$ the average clipping probability is given by

$$
\begin{aligned}
P c_{\alpha} & =\frac{\sum_{n=0}^{N_{v}}\left(n-N_{s}\right)^{+} \times B\left(N_{v}, n, r\right)}{\sum_{n=1}^{N_{v}} n \times B\left(N_{v}, n, r\right)} \\
= & \frac{\sum_{n=N_{s}+1}^{N_{v}}\left(n-N_{s}\right) \times B\left(N_{v}, n, r\right)}{r \times N_{v}} .
\end{aligned}
$$

In (2) the denominator is the expected number of packets generated per frame by all voice stations and the numerator is the expected number of packets which cannot be sent because there are only $N_{s}$ slots available.

\section{B. Upper Bound Clipping Probability}

In the worst case, which determines the upper bound clipping probability, a voice station starting an active period is always the last one to capture the next slot, i.e., it has to wait until there are less than $N_{s}$ active voice stations. For 1PC, this occurs for the last voice station on the bus. Define $P_{i, j}$ as the probability that there will be $j$ active voice stations in the next frame, given that there are a total of $N_{v}-1$ independent voice stations and $i$ of them are active in the current frame. Due to assumptions 2) and 3), the above condition occurs if $n$ of the $i$ active voice stations remain active and $j-n$ of the $N_{v}-1-i$ idle voice stations become active in the next frame, where the lower limit of $n$ is given by the conditions $j-n \leq N_{v}-1-i$ and $n \geq 0$ and the upper limit of $n$ is given by the conditions $i-n \geq 0$ and $j-n \geq 0$. Therefore

$$
\begin{aligned}
P_{i, j}= & \sum_{n=\max \left(0, j+i-N_{v}+1\right)}^{\min (i, j)}\left(\begin{array}{c}
i \\
n
\end{array}\right) \\
& \cdot\left(\begin{array}{c}
N_{v}-1-i \\
j-n
\end{array}\right) q_{t t}^{n} q_{t s}^{i-n} q_{s t}^{j-n} q_{s s}^{N_{v}-1-i-j+n}
\end{aligned}
$$

where $q_{x y}$ is the probability that the state of a station will become $y$ in the next frame given that it is in state $x$ in the current frame, with $x, y=t$ (talk or active) or $s$ (silent or idle). For a frame of period $f, q_{s s}=e^{-f \alpha}, q_{s t}=1-q_{s s}, q_{t t}=e^{-f \beta}$ and $q_{t s}=1-q_{t t}$ where $1 / \alpha$ and $1 / \beta$ are the average idle and active periods of a voice source.

Denote $E_{n}$ as the expected number of additional packets the last voice station clips before capturing a slot when there are $n$ active voice stations upstream. Since the last voice station clips one packet per frame until less than $N_{s}$ voice stations are active, $E_{n}$ is given by the following recursive equation:

$$
E_{n}= \begin{cases}\sum_{i=N_{s}}^{N_{v}-1} P_{n, i} \times\left(1+E_{i}\right)+\sum_{i=0}^{N_{s}-1} P_{n, i}, & n \geq N_{s} \\ 0, & n<N_{s} .\end{cases}
$$

By expressing the above equations in matrix form, as shown in (5) at the bottom of the following page, $E_{n}, n \geq N_{s}$ can be solved as (6) shown at the bottom of the following page 
where $[X]^{-1}$ and $[X]^{T}$ are the inverse and transpose of matrix $[X]$, respectively. The expected number of packets $C_{l}$ the last station clips before capturing a slot is then given by

$$
C_{l}=\sum_{n=N_{s}}^{N_{v}-1} B\left(N_{v}-1, n, r\right) \times E_{n} .
$$

As the average number of packets generated in each talkspurt is $1 /(\beta f)$, the clipping probability of the last station is given by

$$
P c_{l}=C_{l} \times f \times \beta .
$$

\section{Lower Bound Clipping Probability}

In the best case, which determines the lower bound clipping probability and frames to reserve, a voice station starting an active period can always capture the next available slot. For 1PC, this occurs for the first voice station on the bus. Denote $C_{f}$ as the expected number of packets the first station clips before capturing a slot. Due to assumption 1), if the first station becomes active when there are less than $N_{s}$ stations active, no packets will be clipped, otherwise one packet per frame will be clipped until one of the active stations becomes idle and releases a reserved slot. Therefore

$$
\begin{aligned}
C_{f} & =\sum_{n=N_{s}}^{N_{v}-1} B\left(N_{v}-1, n, r\right) \sum_{k=1}^{\infty} k q_{t t}^{N_{s}(k-1)}\left(1-q_{t t}^{N_{s}}\right) \\
& =\sum_{n=N_{s}}^{N_{v}-1} \frac{B\left(N_{v}-1, n, r\right)}{\left(1-q_{t t}^{N_{s}}\right)} .
\end{aligned}
$$

Similar to above, the lower bound clipping probability is

$$
P c_{f}=C_{f} \times f \times \beta .
$$

If a waste-free voice reservation protocol is fair, all voice stations will have the same clipping probability given by (2); otherwise the clipping probability $P c_{j}$ of a voice station $j$ will be between the lower bound and upper bound values as determined above, i.e.,

$$
P_{c_{f}} \leq P c_{j} \leq P c_{l} .
$$

\section{Probability Distribution of Number of Clipped Packets}

To find the number of RA slots required to satisfy the Campanella criteria we need to obtain the probability distribution of the number of packets being clipped in a talkspurt. This distribution is dependent on the nature of the capturing process and is difficult to evaluate in general. To facilitate the later analysis, we adopt a first-come first-served (FCFS) assumption. It can be seen later that the approximations are in good agreement with the simulation results when the number of RA slots is large.

Consider that when voice station $X$ becomes active in frame $k$ it joins a FCFS queue for capturing a slot and that assumptions 1)-4) above are still valid. Let $N_{t}$ and $N_{q}$ denote the numbers of voice stations that are active and waiting in the queue, respectively, when $X$ joins the queue in frame $k$. Obviously, $N_{q}=\left(N_{t}-N_{s}\right)^{+}$where $N_{s}$ is the number of RA slots. $P_{w}\left(N_{c} \mid N_{t}\right)$, the probability that $X$ clips $N_{c}$ packets before capturing a slot (i.e., $X$ captures a slot in the $k+N_{c}+1$ th frame) given that there are $N_{t}$ active stations when $X$ arrives at the queue, is given by

$$
P_{w}\left(N_{c} \mid N_{t}\right)=\left\{\begin{array}{l}
1, \quad N_{c}=0, N_{t}<N_{s} \\
\left(\begin{array}{c}
N_{c}-1 \\
N_{q}
\end{array}\right) Q_{t}^{N_{q}+1}\left(1-Q_{t}\right)^{N_{c}-N_{q}-1} \\
N_{c} \geq N_{q}+1, N_{t} \geq N_{s} \\
0, \quad \text { otherwise }
\end{array}\right.
$$

where $Q_{t}=\left(1-q_{t t}^{N_{s}}\right)$.

Equation (12) means that $X$ can capture a slot immediately if there are less than $N_{s}$ active voice stations when it arrives at the queue, otherwise the number of packets it clips is given by the sum of the numbers of packets clipped by $N_{q}+1$ stations (including $X$ ) while each station is at the head of the queue, which probability distribution is given by the $\left(N_{q}+1\right)$-fold convolution of the geometrical distribution $G(w)=Q_{t}\left(1-Q_{t}\right)^{w-1}$ of the number of packets clipped by the station at the head of the queue before it captures a slot. Note that as the minimum number of packets clipped by each station in the queue is one, there are some invalid situations which results in the zero probability in (12). Averaging over $N_{t}$ the probability that $X$ clips $N_{c}$ packets before capturing

$$
\left[\begin{array}{c}
E_{N s} \\
E_{N_{s}+1} \\
\vdots \\
E_{N_{v}-1}
\end{array}\right]=\left[\begin{array}{cccc}
P_{N_{s}, N_{s}} & P_{N_{s}, N_{s}+1} & \cdots & P_{N_{s}, N_{v}-1} \\
P_{N_{s}+1, N_{s}} & P_{N_{s}+1, N_{s}+1} & \cdots & P_{N_{s}+1, N_{v}-1} \\
\vdots & \vdots & \vdots & \vdots \\
P_{N_{v}-1, N_{s}} & P_{N_{v}-1, N_{s}+1} & \cdots & P_{N_{v}-1, N_{v}-1}
\end{array}\right] \cdot\left[\begin{array}{c}
E_{N_{s}} \\
E_{N_{s}+1} \\
\vdots \\
E_{N_{v}-1}
\end{array}\right]+\left[\begin{array}{c}
1 \\
1 \\
\vdots \\
1
\end{array}\right]
$$

$$
\left[\begin{array}{llll}
E_{N_{s}} & E_{N_{s}+1} & \cdots & E_{N_{v}-1}
\end{array}\right]=\left[\begin{array}{l}
1 \\
\vdots \\
1
\end{array}\right]^{T}\left[\begin{array}{cccc}
1-P_{N_{s}, N_{s}} & -P_{N_{s}+1, N_{s}} & \cdots & -P_{N_{v}-1, N_{s}} \\
-P_{N_{s}, N_{s}+1} & 1-P_{N_{s}+1, N_{s}+1} & \cdots & -P_{N_{v}-1, N_{s}+1} \\
\vdots & \vdots & \vdots & \vdots \\
-P_{N_{s}, N_{v}-1} & -P_{N_{s}+1, N_{v}-1} & \cdots & 1-P_{N_{v}-1, N_{v}-1}
\end{array}\right]^{-1}
$$


a slot is given by

$$
P_{w}\left(N_{c}\right)=\sum_{N_{t}=0}^{N_{v}-1} P\left(N_{c} \mid N_{t}\right) B\left(N_{v}-1, N_{t}, r\right) .
$$

The probability that station $X$ clips at most $N_{c}$ packets before capturing a slot is

$$
P_{w}\left(\leq N_{c}\right)=\sum_{j=0}^{N_{c}} P_{w}\left(N_{c}\right) .
$$

For evaluation of the Campanella criteria, we need to find the probability that the clip duration is more than $50 \mathrm{~ms}$, which corresponds to the clipping 8.3 packets. Assuming that linear interpolation can be used, the required probability is given by

$$
P_{w}(>8.3)=1-\left\{P_{w}(\leq 8)+0.3 \times\left[P_{w}(\leq 9)-P_{w}(\leq 8)\right]\right\} .
$$

\section{E. Utilization}

When there are $n<N_{s}$ voice stations active the utilization is $n / N_{s}$, otherwise it is equal to one. Hence, the overall average utilization is

$$
U=\sum_{n=1}^{N_{s}-1} \frac{n \times B\left(N_{v}, n, r\right)}{N_{s}}+\sum_{n=N_{s}}^{N_{v}} B\left(N_{v}, n, r\right) .
$$

\section{Discussions on Simulation and Analytical Results}

A simulation program was written in Simscript II.5 to generate results for comparison with the above analytical calculations. Unless otherwise specified, the simulation parameters follow the voice system model given in Section II (i.e., 50 transmitting MAN nodes on bus A running at $155 \mathrm{Mb} / \mathrm{s}$ with one voice station per node each engaged in a call throughout the time period considered). The distance between adjacent nodes is equivalent to ten slots, and 300000 frames were sent for each simulation. Note that running simulations for RA access is very time consuming due to its reservation nature. This is because once a reservation is secured, isochronous service is provided and no packet losses occur (or the traffic condition is basically unchanged). That means we have to simulate a huge number of talkspurts in order to obtain steady state results. Therefore it is difficult to run simulations with a very large number of voice sources. Nevertheless the simulated MAN, with a spanning distance of 500 slots (round trip delay of 1000 slots) does cover a realistic city area and can be made contention free as shown above. Results are presented and discussed below.

\section{A. Clipping Probability}

Fig. 3 shows the clipping probability variations for both $1 \mathrm{PC}$ and CC. It can be seen that $1 \mathrm{PC}$ is unfair but $\mathrm{CC}$ provides reasonably fair service consistent with the analytical calculations as shown by the solid lines. Note that the circumference of the simulated MAN is about $560 \mathrm{~km}$, which well exceeds the expected coverage area of most cities. The results therefore indicate the propagation delay in a practical MAN should
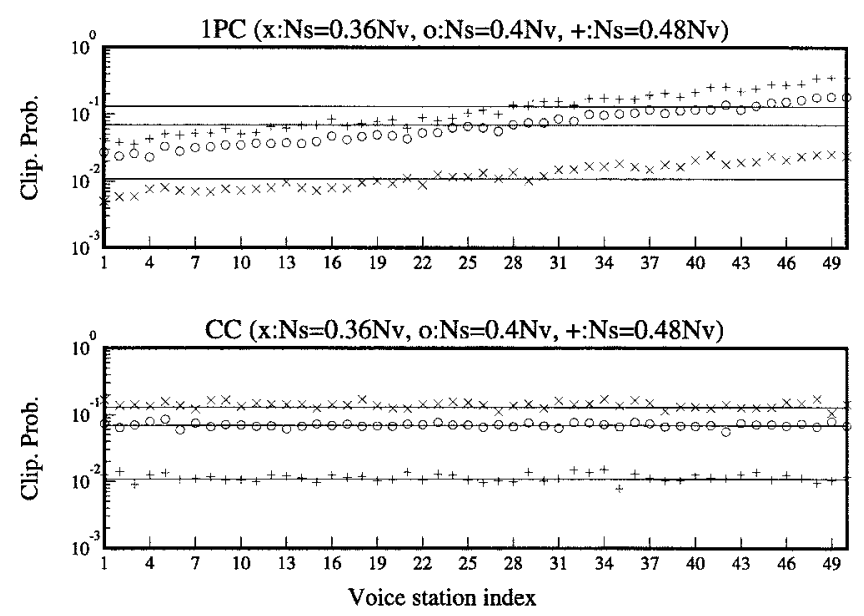

Fig. 3. Clipping probability variations along the MAN bus.

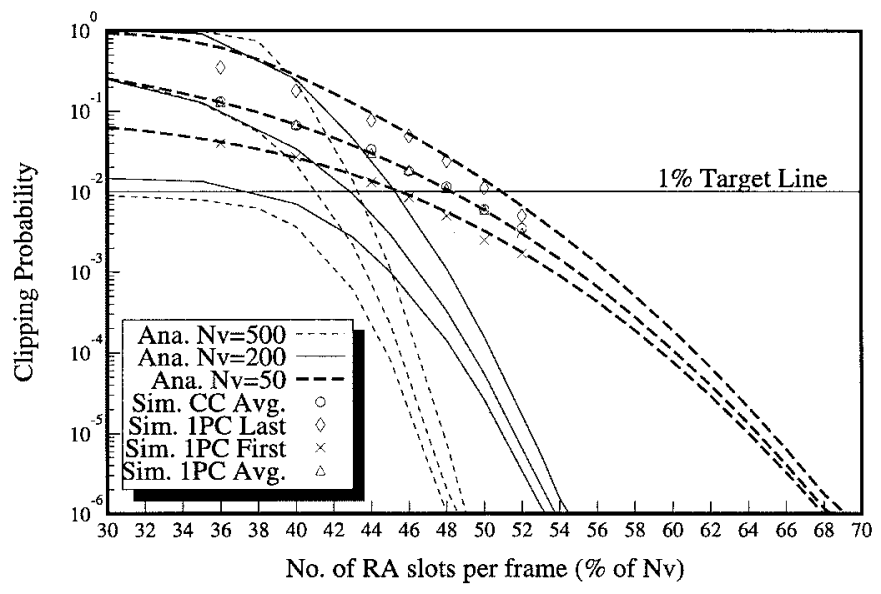

Fig. 4. Upper bound, lower bound, and average clipping probabilities.

not cause any access fairness problem for RA access with CC. Fig. 4 shows the simulation results of average clipping probabilities in comparison with the analytical calculations. In the case of 1PC, the clipping probabilities for the first station (lower bound) and the last station (upper bound) are also shown. The close agreement between the analytical results and the simulation results lends confidence to the correctness of both models. As expected, in the case of 1PC, the last voice station experiences the largest clipping probability. The difference in performance between the first and last stations diminishes, however, when the number of RA slots increases, indicating that the fairness problem could be lessened. For $\mathrm{CC}$, it is found that all stations have nearly the same clipping probabilities consistent with the analytical calculations. With CC and 1PC, about $24 \mathrm{RA}$ slots (48\% of $N_{v}$ ) and $25 \mathrm{RA}$ slots ( $50 \%$ of $N_{v}$ ), respectively, are required to ensure that all 50 voice stations satisfy the clipping probability criteria. When $N_{v}$ is increased to 200 and 500, the statistical multiplexing gain is substantially increased, and the average upper bound and lower bound clipping probabilities drop dramatically for the same percentage of $N_{s}$ relative to $N_{v}$. Therefore, when the number of voice stations is large, the required percentage of RA slots to meet the clipping probability criteria approaches 


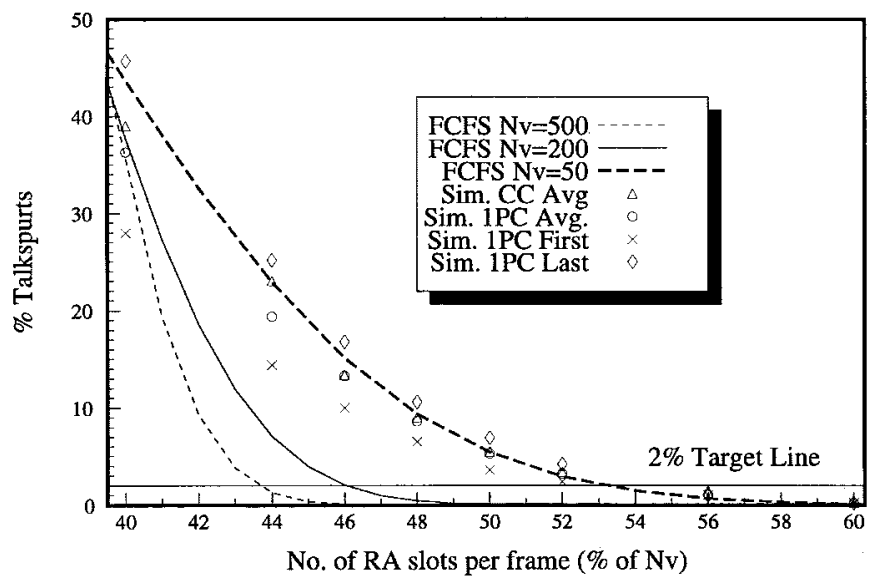

Fig. 5. Percentage of talkspurts having clip durations greater than $50 \mathrm{~ms}$.

the voice activity factor of $40 \%$, resulting in a multiplexing gain of 2.5 as compared to PA access.

\section{B. Campanella Criteria}

Fig. 5 shows the simulation results for the Campanella criteria in comparison with that calculated from (15). In both the case of 1PC and CC, the average percentages of talkspurts having clip duration of over $50 \mathrm{~ms}$ are close to that predicted by the FCFS model when the number of RA slots is large. While 1PC is unfair, the results for the first and last stations (hence all other stations) tend to converge to the analytical results as the number of RA slots per frame increases. With CC and 1PC, about 27 (54\% of $N_{v}$ ) and 28 RA slots ( $56 \%$ of $N_{v}$ ), respectively, are sufficient to ensure that all 50 voice stations can satisfy the Campanella criteria. When $N_{v}$ is increased to 200 and 500, the percentage of RA slots required to satisfy the Campanella criteria decreases dramatically and approaches the voice activity factor of $40 \%$.

\section{Utilization}

Fig. 6 shows that the simulation results of utilization for both 1PC and CC are nearly the same and agree closely with those calculated using (16). With $30 \mathrm{RA}$ slots for 50 voice stations, the utilization is about $68 \%$ as compared to $40 \%$ for PA access. With $N_{v}=200$ and 500 , the utilization increases when the percentage number of RA slot is small but converges to that of $N_{v}=50$ when the percentage is large. This indicates that utilization is apparently independent of the number of voice stations when the number of RA slots is large. Nevertheless, as the number of voice stations increases, fewer RA slots are required per voice station to achieve the clipping probability objective; hence the protocol is more efficient and utilization approaches $100 \%$.

\section{Simulation Using a Real Voice Conversation}

To assess the actual quality of a voice conversation transported by RA access with 1PC, we simulated the transmissions of a real speech signal by the fiftieth voice station while keeping the other 49 stations to the original two-state voice model. We use 1PC because we want to test the worst case

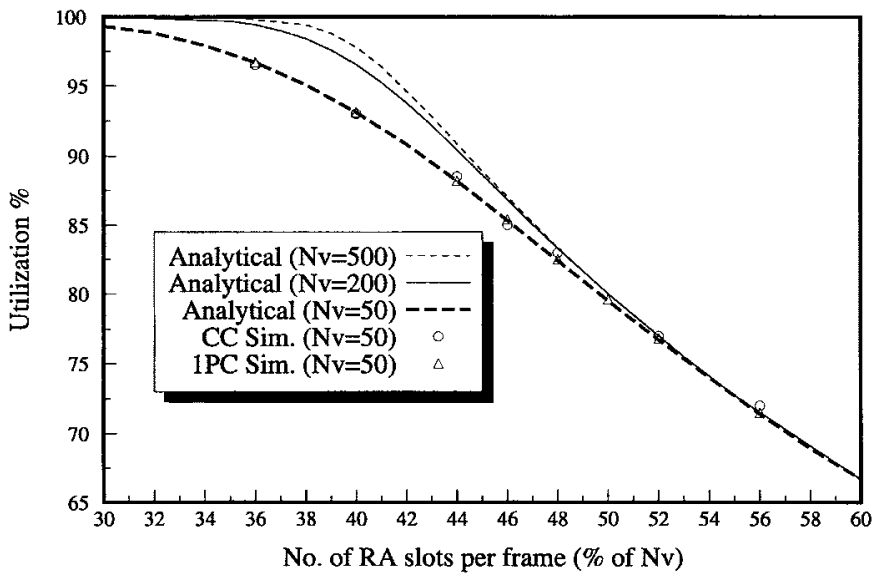

Fig. 6. Slot utilization.

behavior. The speech signal was produced by recording $20 \mathrm{~s}$ of one side of a telephone conversation. The talkspurt and silent intervals were then determined as follows: 1) talk to silent transition occurs when the signal amplitude stays within the background noise envelope, which is found by averaging all the positive peak and negative peak background noise voltages during the silent periods, for over $200 \mathrm{~ms}$ [14] and 2) silent to talk transition occurs if the amplitude falls outside the background noise envelope. During the talkspurt periods, the voice signal was sampled at 8000 samples per second with 8 bits per sample giving $64 \mathrm{~Kb} / \mathrm{s} .48$ samples were then grouped to form one voice packet every $6 \mathrm{~ms}$ which was transported by the RA access protocol. The received signal was then regenerated from the successfully received packets. Although there is no voice activity during the silent intervals, it may still be desirable to maintain a certain background noise level for the listener. We assume that this can be done by transmitting an extra frame of noise level at the end of each talkspurt and the receiver repeats it to the listener. In other words, the silent gaps and missing samples are filled with background noise instead of complete silence. The simulation time was 100000 frames during which the $20 \mathrm{~s}$ of speech samples were repeated continuously to find the worst situation (i.e., when the number of clipped packets is largest). Fig. 7 shows the worst regenerated signals for $N_{s}=20,25$, and 30 . It is apparent that, when $N_{s}=20$ the speech is totally unrecognizable. When $N_{s}$ is increased to 25 , the quality is greatly improved. However, one word was missed. With $N_{s}=30$ very few packets were clipped and no noticeable degradation was found from the regenerated speech signal. This test indicates that even with 1PC, 25 slots should be marginally acceptable and 30 slots can provide very satisfactory service which is consistent with the above analysis.

\section{E. Call Admission Control}

The purpose of call admission control is to decide whether a new voice call can be admitted without affecting the quality of service of existing voice calls. In general, the analytical calculations in Section IV can be applied for call admission control. Here we assume that CC is adopted for RA access and (2) is used for call admission control because of its simplicity 

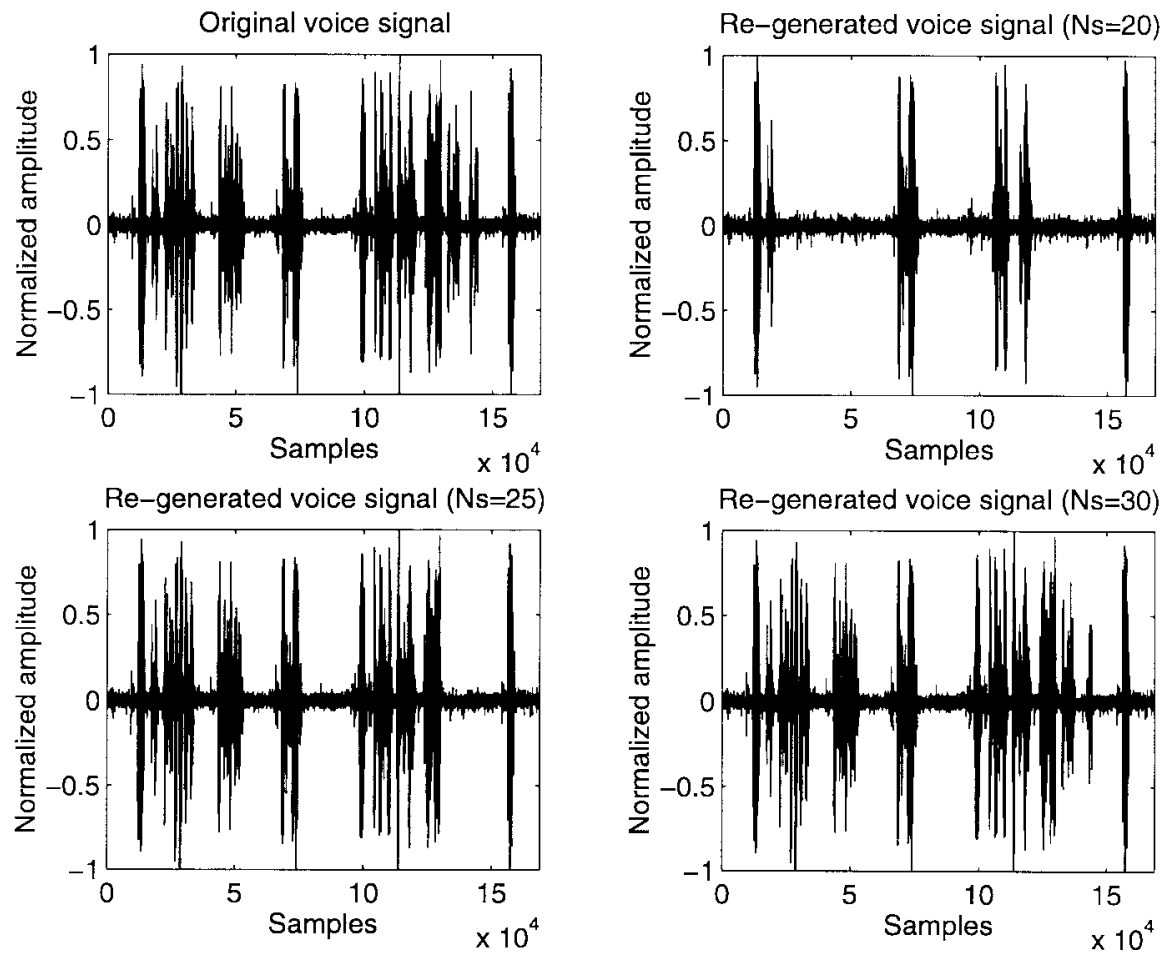

Fig. 7. Simulation using a real speech signal.

and computational efficiency. In fact, by choosing a proper target clipping probability $P_{\text {target }}$ we can also accommodate the Campanella criteria by using (2). This is because for a clipping probability of $P_{\text {target }}$ it already guarantees that more than or equal to $1-\left(P_{\text {target }} / \beta f\right)$ of the talkspurts are completed without any packet loss. This can be shown easily by noting that if fraction $x$ of the talkspurts are completed without any packet loss, the rest $(1-x)$ must have lost at least one packet each, giving a lower bound clipping probability of $(1-x) \times \beta f$ where $1 / \beta f$ is the expected number of packets generated by a station during each talkspurt. That means if we set $P_{\text {target }}$ such that $\left(1-\left(P_{\text {target }} / \beta f\right)\right)>98 \%$ more than $98 \%$ of talkspurts will have no packet loss, which is obviously a more stringent requirement than the Campanella criteria. To meet this stringent criteria, $P_{\text {target }}$ must not be more than 0.00008 . Fig. 8 shows the maximum number of voice calls $N_{v_{\max }}$ that can be admitted into the network against the assigned voice bandwidth for the following:

- clipping probability criteria $-P_{\text {target }}=0.01$;

- stringent criteria- $P_{\text {target }}=0.00008$.

The results were found by finding the maximum value of $N_{v}$ which satisfies the following inequality using the bisection method:

$$
\frac{\sum_{n=N_{s}+1}^{N_{v}}\left(n-N_{s}\right) \times B\left(N_{v}, n, r\right)}{r \times N_{v}}<P_{\text {target }} .
$$

The graphs also show the results for the case of PA access (i.e., $N_{v_{\max }}=N_{s}$ ) and of ideal statistical multiplexing (i.e., $\left.N_{v_{\max }}=N_{s} / r\right)$. Note that the former and latter equations give the lower bound (i.e., worst case) and upper bound (i.e.,

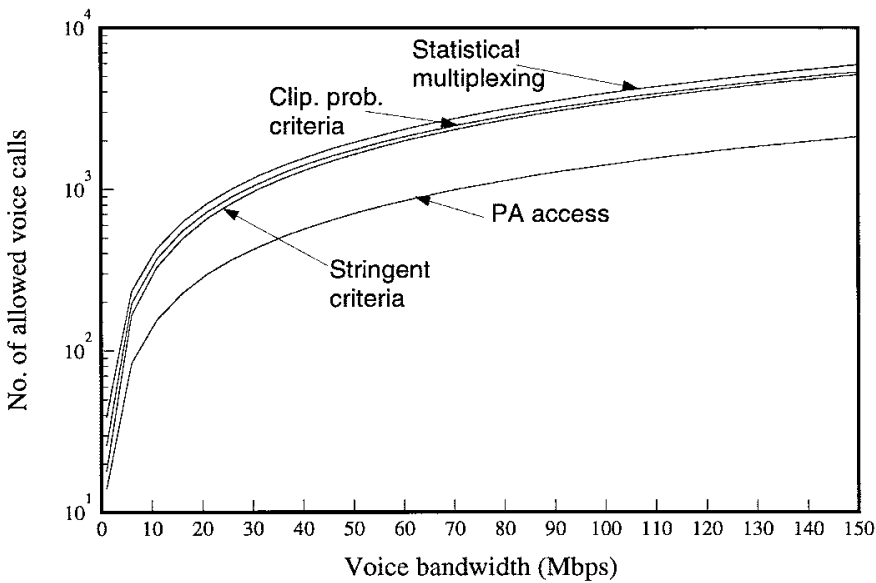

Fig. 8. Number of allowed voice calls for different voice bandwidth (RA access with CC).

best case) results, respectively. It can be seen from the figure that RA access supports significantly more voice calls than PA access (the conventional circuit switching approach). Even with the stringent admission criteria, the number of voice calls that can be admitted is close to that of the ideal results. Although QA access should provide comparable results, it suffers from the well-known unfairness problem and cannot provide isochronous transport service so RA access with CC should be a more preferable method.

\section{CONCLUSIONS}

We have proposed a novel RA access technique for efficient voice communications over dual-bus MAN's/LAN's. In combination with a new $\mathrm{CC}$ mechanism, RA access provides 
fair and isochronous voice transport service while allowing statistical multiplexing. In fact, with sufficient RA slots, a voice station starting a talkspurt can reserve an RA slot almost immediately indicating that service close to PA access can be provided. We have also derived an analytical model for general waste-free voice reservation systems. This model can be used for analyzing RA access and may also be adapted for other packet voice systems. By means of theoretical analyses and computer simulations, we have quantified the performance of the RA access protocol. The analytical and simulation results are further validated by a simulation using real voice samples to assess the actual voice quality. Our results indicate that RA access can provide a multiplexing gain of 1.7 over PA access while maintaining an acceptable voice quality. As the number of voice stations increases, the multiplexing gain approaches the theoretical limit of 2.5. Important applications of RA access are to facilitate voice transport over SMDS networks, MAN-based wireless personal communication networks, and other ATM/MAN based systems.

\section{REFERENCES}

[1] IEEE Standard for LAN's and MAN's: Distributed Queue Dual Bus (DODB) Subnetwork of a MAN, 802.6, Dec. 1990.

[2] G. C. Kessler, ISDN, 2nd ed. New York: McGraw-Hill, 1993, ch. 11

[3] W. R. Byrne, G. W. R. Luderer, G. Clapp, B. L. Nelson, and H. J. Kafka, "Evolution of metropolitan area networks to broadband ISDN," IEEE Commun. Mag., vol. 29, Jan. 1991, pp. 69-82.

[4] J. Filipiak, "Shaping internetworking MAN's into an evolutionary BISDN," Comput. Networks ISDN Syst., vol. 20, pp. 343-349, 1990.

[5] D. J. Goodman, "Cellular packet communications," IEEE Trans. Commun., vol. 38, pp. 1272-1280, Aug. 1990.

[6] A. D. Malyan, L. J. Ng, R. W. Donaldson, and V. C. M. Leung, "Network architecture and signalling for wireless personal communications," IEEE J. Select. Areas Commun., vol. 11, pp. 830-841, Aug. 1993.

[7] D. J. Goodman, R. A. Valenzuela, K. T. Gayliard, and B. Ramamurthi, "Packet reservation multiple access for local wireless communications," IEEE Trans. Commun., vol. 37, pp. 885-890, Aug. 1989.

[8] K. Apostolidis, L. F. Merakos, and X. H. Xing, "A reservation protocol for packet voice and data integration in unidirectional bus network," IEEE Trans. Commun., vol. 41, pp. 478-485, Mar. 1993.

[9] J. O. Limb and L. E. Flamm, "A distributed local area network packet protocol for combined voice and data transmission," IEEE J. Select. Areas Commun., vol. SAC-1, pp. 926-934, Nov. 1983.

[10] B. T. Doshi, S. Dravida, P. D. Magill, C. A. Siller, Jr., and K. Sriram, "A broadband multiple access protocol for STM, ATM, and variable length data services on hybrid fiber-coax networks," Bell Labs Tech. J., vol. 1 , no. 1 , pp. 36-65, 1996.

[11] B. Mukherjee and J. S. Meditch, "Integrating voice with the pipersistent protocol for unidirectional broadcast bus networks," IEEE Trans. Commun., vol. 36, pp. 1287-1295, Dec. 1988.

[12] K. Sriram, P. K. Varshney, and J. G. Shanthikumar, "Discrete time analysis of integrated voice/data multiplexers with and without speech activity detectors," IEEE J. Select. Areas Commun., vol. SAC-1, pp. 1124-1132, Dec. 1983.

[13] J. K. Choi, J. U. Seo, and C. K. Un, "Performance analysis of a packetswitched synchronous voice/data transmission system," IEEE Trans. Commun., vol. 38, pp. 1419-1429, Sept. 1990.

[14] P. T. Brady," A model for generating on-off speech patterns in two way conversation," Bell Sys. Tech. J., vol. 48, pp. 2445-2472, Sept. 1969.

[15] B. Maglaris, D. Anastassiou, P. Sen, G. Karlson, and J. Robbins, "Performance models of statistical multiplexing in packet video communications," IEEE Trans. Commun., vol. 36, pp. 834-844, July 1988.
[16] J. G. Gruber and N. H. Le, "Performance requirements for integrated voice/data networks," IEEE J. Select. Areas Commun., vol. SAC-1, pp. 981-1004, Dec. 1983.

[17] S. J. Campanells, "Digital speech interpolation," COMSAT Tech. Rev., vol. 6, pp. 127-158, Spring 1976.

[18] H. C. B. Chan and V. C. M. Leung, "Performance analysis of reservation arbitrated access for statistical multiplexing voice traffic over dual bus metropolitan area networks," in Proc. Globecom'95, Singapore, Nov. 1995, pp. 675-679.

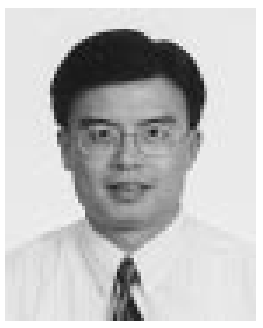

Henry C. B. Chan (M'98) received the B.A. and M.A. degrees from the University of Cambridge, Cambridge, U.K., in 1988 and 1992, respectively, and the Ph.D. degree from the University of British Columbia, Vancouver, Canada in 1997

Between 1988 and 1993, he worked with Hong Kong Telecom, Hong Kong, primarily on the development of data communication services. From 1989 to 1993 , he was a Visiting Evening Lecturer at the Hong Kong Polytechnic. During his graduate studies, he was a Research and Teaching Assistant in the Communication Research Group of the University of British Columbia. Between October 1997 and August 1998, he was with BC Tel Advanced Communications working on the development of high-speed networking technologies and ATM-based services. During the same period, he was also a part-time Post-Doctoral Research Fellow at the University of British Columbia. Currently, he is an Assistant Professor in the Department of Computing, Hong Kong Polytechnic University, Kowloon. His research interests include high-speed networks, Internet technologies, and wireless ATM.

Dr. Chan was awarded an NSERC Postgraduate Scholarship from the Canadian Government during his graduate studies at the University of British Columbia, a University of British Columbia Graduate Fellowship, and a BC TEL Graduate Scholarship. He was the winner of the IEE (HK) Younger Member Paper Contest in 1992. He is a member of ACM and the Chartered Institute of Marketing.

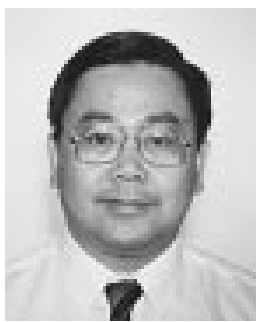

Victor C. M. Leung (S'75-M'79-SM'97) received the B.A.Sc. (Hons.) and Ph.D. degrees in electrical engineering from the University of British Columbia, Vancouver, British Columbia, in 1977 and 1981, respectively.

From 1981 to 1987, he was a Senior Member of Technical Staff at Microtel Pacific Research Limited, specializing in the planning, design, and analysis of satellite communication systems. In 1986 and 1987 he was a part-time Visiting Assistant Professor at Simon Fraser University, Burnaby, British Columbia. In 1988, he was a Lecturer in the Department of Electronics at the Chinese University of Hong Kong, Hong Kong. He joined the Department of Electrical Engineering at the University of British Columbia in 1989, where he is an Associate Professor and a Member of the Centre for Integrated Computer Systems Research. He is also a Project Leader at the Canadian Institute for Telecommunications Research, a Network of Centres of Excellence funded by the Canadian Government. His research interests are in the areas of architectural and protocol design and performance analysis for computer and telecommunication networks, with applications in satellite, mobile, personal communications, and high-speed networks.

Dr. Leung was awarded the APEBC Gold Medal as the head of the graduating class in the Faculty of Applied Science and a Canadian Natural Sciences and Engineering Research Council Postgraduate Scholarship. He is a voting member of ACM. 